\title{
Borboletas (Lepidoptera: Papilionoidea e Hesperioidea) de Matas Paludosas e Matas de Restinga da Planície Costeira da região Sul do Brasil
}

\author{
Juliane Bellaver ${ }^{1,3}$, Cristiano Agra Iserhard ${ }^{2}$, Jessie Pereira dos Santos ${ }^{2}$,Ana Kristina Silva ${ }^{2}$, \\ Márcio Torres ${ }^{I}$, Ricardo Russo Siewert ${ }^{1}$, Alfred Moser ${ }^{I}$ \& Helena Piccoli Romanowski ${ }^{I}$ \\ ${ }^{1}$ Programa de Pós-graduação em Biologia Animal, Departamento de Zoologia, Universidade Federal do \\ Rio Grande do Sul-UFRGS, Av. Bento Gonçalves, 9500, Prédio 43435, Laboratório 218, CEP 91501-970, \\ Porto Alegre, RS, Brasil. www.ufrgs.br/zoologia \\ ${ }^{2}$ Departamento de Biologia Animal, Instituto de Biologia, Universidade Estadual de \\ Campinas - UNICAMP, Cidade Universitária Zeferino Vaz, s/n, Barão Geraldo, CP 6109, \\ CEP 13083-970, Campinas, SP, Brasil. www.ib.unicamp.br \\ ${ }^{3}$ Autor para correspondência: Juliane Bellaver, e-mail: jubellaver@yahoo.com.br
}

BELLAVER, J., ISERHARD, C.A., SANTOS, J.P., SILVA, A.K., TORRES, M., SIEWERT, R.R., MOSER, A. \& ROMANOWSKI, H.P. Butterflies (Lepidoptera: Papilionoidea and Hesperioidea) from Swamp forests and Restinga forests at the southern Brazilian Coastal Plain. Biota Neotrop. 12(4): http://www.biotaneotropica. org.br/v12n4/en/abstract?inventory+bn01812042012

Abstract: This paper presents a butterfly species list of Swamp and Resting forests in the Coastal Plain of Rio Grande do Sul and Santa Catarina States aiming to contribute to the knowledge of butterflies for these environments in the Atlantic Forest. Data compilation was obtained through inventories carried out in the years 2005 and 2011 with two sampling protocols (bait traps and butterfly nets). After 10.920 trap-hours and 360 net-hours 225 species of butterflies were recorded belonging to six families and 19 subfamilies. Twenty-five species are new records for the Rio Grande do Sul State and 35 species are new records for the Atlantic Forest in Rio Grande do Sul, with six Nymphalidae, ten Hesperiidae, twelve Lycaenidae and seven Riodinidae. The results obtained in the present study are fundamental for the knowledge and conservation of the taxa studied and to their associated habitats. Keywords: Atlantic Forest, Itapeva State Park, new records, species richness.

BELLAVER, J., ISERHARD, C.A., SANTOS, J.P., SILVA, A.K., TORRES, M., SIEWERT, R.R., MOSER, A. \& ROMANOWSKI, H.P. Borboletas (Lepidoptera: Papilionoidea e Hesperioidea) de Matas Paludosas e Matas de Restinga da Planície Costeira da região Sul do Brasil. Biota Neotrop. 12(4): http://www.biotaneotropica. org.br/v12n4/pt/abstract?inventory+bn01812042012

Resumo: Este trabalho teve como objetivo elaborar uma lista de espécies de borboletas de Matas Paludosas e de Restingas da Planície Costeira do Rio Grande do Sul e de Santa Catarina contribuindo para o conhecimento da fauna de borboletas da Mata Atlântica. Foram compilados dados obtidos com amostragens realizadas através de duas técnicas de coleta (armadilha com isca atrativa e rede entomológica) nos anos de 2005 e 2011. Com um esforço amostral de 10.920 horas com armadilhas e de 360 horas-rede foram encontradas 225 espécies de borboletas nos dois ambientes, distribuídas em seis famílias e 19 subfamílias. Vinte e cinco espécies são registros novos ainda não publicados para o Rio Grande do Sul e 35 espécies são novos registros para a Mata Atlântica do Rio Grande do Sul, sendo seis Nymphalidae, dez Hesperiidae, doze Lycaenidae e sete Riodinidae. Os resultados gerados no presente estudo são fundamentais para o conhecimento e conservação dos táxons estudados bem como dos ambientes aos quais estão associados.

Palavras-chave: Mata Atlântica, Parque Estadual de Itapeva, novos registros, riqueza de espécies. 


\section{Introdução}

A aceleração do processo de destruição da flora e fauna em diferentes biomas nas últimas décadas tem incrementado a necessidade na conservação de habitats naturais e, principalmente, em maiores esforços para o conhecimento da biodiversidade. Entretanto, o tempo disponível para a geração deste conhecimento é limitado (Daily \& Ehrlich 1995, Hughes et al. 2000). Trabalhos de monitoramento e planejamento em conservação são mais efetivos quando são embasados por um conhecimento prévio da ocorrência dos organismos em um determinado local (Colombo et al. 2008). Tais informações são obtidas através da realização de inventários de diversidade (e.g. Freitas et al. 2003, Iserhard \& Romanowski 2004), sendo importantes fontes de informação para a restauração, manutenção da composição, estrutura e funcionamento das comunidades naturais (Brown \& Freitas 2000a, Freitas et al. 2006, Bond-Buckup 2008, Santos et al. 2008).

Um dos grupos de invertebrados mais conhecidos e que vem aumentando gradativamente seu uso em monitoramento e avaliação ambiental são as borboletas (Brown 1991, New 1997), pois respondem rapidamente às perturbações nos sistemas pela íntima associação com seus microhábitats e por serem especialistas em recursos específicos no ambiente (Brown 1997, Freitas et al. 2003). Além disso, são abundantes na região Neotropical e possuem alta diversidade com cerca de 7800 espécies descritas (Lamas 2004), sendo que no Brasil estima-se que ocorram em torno de 3300 espécies (Brown \& Freitas 1999). Para o sul do Brasil, Morais et al. (2007) citam 679 espécies registradas na literatura para o Rio Grande do Sul. Dados atualizados, todavia, indicam a ocorrência de, pelo menos, mil espécies no Estado (Romanowski, dados não publicados).

O bioma Mata Atlântica estava originalmente distribuído em uma extensa área ao longo da região costeira do Brasil. Atualmente, restam em torno de 11 a $16 \%$ de sua extensão original, sendo que a maioria de seus remanescentes estão distribuídos em pequenas e isoladas manchas florestais (Ribeiro et al. 2009). As Matas de Restinga fazem parte do bioma Mata Atlântica e são definidas como a faixa de vegetação que ocorre em áreas planas e arenosas localizadas, de uma maneira ampla, entre o oceano e as serras (Waechter 1985). Embora estejam localizadas junto a áreas com alta densidade humana, carecem de informação científica (Rocha et al. 2004) com elevadas taxas de conversão em sistemas antrópicos. As Matas Paludosas (Floresta Ombrófila Densa das Terras Baixas) localizam-se em regiões litorâneas próximas ao mar e caracterizamse por apresentar alagamento permanente ou temporário com solo lodoso e rico em matéria orgânica vegetal (Rambo 2005). Conservam uma importante biodiversidade e um grande número de espécies ameaçadas (Rio Grande do Sul, 2006). Embora as Matas Paludosas possuam uma distribuição naturalmente fragmentada (Toniato et al. 1998, Paschoal \& Cavassan 1999), o intenso impacto de agropecuária e de especulação imobiliária, que só aumentou nos últimos anos, tornou-as limitadas em reduzidos fragmentos com amplas áreas de matriz circundantes, compondo uma conformação amplamente modificada da paisagem original.

Nos últimos doze anos, inventários de borboletas na Mata Atlântica do Rio Grande do Sul foram intensificados, principalmente no que diz respeito à Floresta Ombrófila Densa na encosta do Planalto Basáltico (Iserhard \& Romanowski 2004, Iserhard 2009, Santos et al. 2011), na Floresta Ombrófila Mista e nos Campos de Cima da Serra (Teston \& Corseuil 1999, 2000, 2002, Corseuil et al. 2004, Romanowski et al. 2009, Iserhard et al. 2010a, Pedrotti et al. 2011, Ritter et al. 2011, Santos et al. 2011). Porém, estudos abrangendo à Mata de Restinga e à Floresta Ombrófila Densa junto à Planície Costeira norte do Estado ainda são escassos. Os trabalhos já publicados neste ecossistema referem-se a (i) um inventário das borboletas da família Nymphalidae em dez municípios no litoral norte (Quadros et al. 2004) e (ii) à ampliação de distribuição da borboleta Heliconius sara apseudes (Hübner, 1813) na Mata Atlântica (Iserhard et al. 2010b), relativa a inventários de borboletas realizados na planície, encosta e altitude deste bioma no RS.

O presente trabalho teve como objetivo (i) elaborar uma lista de espécies de borboletas de diferentes ambientes de Mata Paludosa e Mata de Restinga em uma ampla área na Planície Costeira norte do Rio Grande do Sul e uma área entre este Estado com Santa Catarina, visando contribuir para o conhecimento da fauna de borboletas através do registro de novas ocorrências para a Mata Atlântica do Rio Grande do Sul.

\section{Material e Métodos}

\section{1. Área de estudo}

Foram estudados dez fragmentos de Mata Paludosa e três áreas de Mata de Restinga em uma região localizada na Planície Costeira norte do Rio Grande do Sul nos municípios de Torres, Morrinhos do Sul, Mampituba e Dom Pedro de Alcântara e Passo de Torres, em Santa Catarina (Figura 1). As Matas de Restinga localizavam-se junto a dunas fixas com vegetação psamófila arbustiva e arbórea, e as Matas Paludosas caracterizavam-se por serem formadas por processo de sucessão vegetal em lagoas, portanto alagadiças, constituídas tanto por áreas de mata em regeneração quanto por floresta com dossel entre 8-12 $\mathrm{m}$ de altura (Colombo et al. 2008). O clima da região é subtropical com verões e invernos relativamente amenos (Maluf 2000). A temperatura anual varia com médias máximas de $29{ }^{\circ} \mathrm{C} \mathrm{a}$ $18^{\circ} \mathrm{C}$ no verão e com médias mínimas de $22^{\circ} \mathrm{C}$ a $11^{\circ} \mathrm{C}$ no inverno; a precipitação anual fica em torno de $1.600 \mathrm{~mm}$ (Agritempo 2012).

\section{Amostragem}

Foram realizadas amostragens através de duas técnicas de coleta: (i) armadilhas com isca atrativa para borboletas frugívoras da família Nymphalidae e (ii) rede entomológica para as borboletas das superfamílias Papilionoidea e Hesperioidea.

Para a primeira técnica foi amostrado um fragmento de Mata Paludosa e três áreas de Mata de Restinga pertencentes ao Parque Estadual de Itapeva (PEVA) em janeiro, maio e novembro de 2005. Posteriormente, foram realizadas duas amostragens (janeiro e março de 2011) em dez fragmentos de Mata Paludosa (incluindo o fragmento do PEVA).

No ano de 2005, em cada área estudada foram colocadas três armadilhas formando uma Unidade Amostral (UA), nas quais, as armadilhas ficavam distantes, aproximadamente, $50 \mathrm{~m}$ uma da outra. Estas permaneceram instaladas durante 48 horas na Mata Paludosa e outras 48 horas na Mata de Restinga perfazendo 360 horas de esforço amostral total. Nas amostragens do ano de 2011, em cada fragmento, duas UAs foram delimitadas, sendo uma na borda e uma no interior. Cada UA era constituída por dois grupos de três armadilhas cada, as quais ficavam distantes em torno de cinco metros entre si. Cada grupo de armadilhas foi disposto em duas transecções lineares, cada uma com $50 \mathrm{~m}$ de extensão entre a borda e o interior, separadas por $100 \mathrm{~m}$ entre si. A isca utilizada foi elaborada através da mistura de banana amassada com caldo de cana fermentada por 48 horas, sendo substituída a cada revisão. As armadilhas foram colocadas na manhã do primeiro dia de amostragem e permaneceram instaladas por mais três dias. O processo de revisão foi realizado a cada 48 horas (totalizando 10.560 horas de esforço amostral).

As amostragens com redes entomológicas foram realizadas em 2005 (nos mesmos meses e nas mesmas ocasiões amostrais descritas 


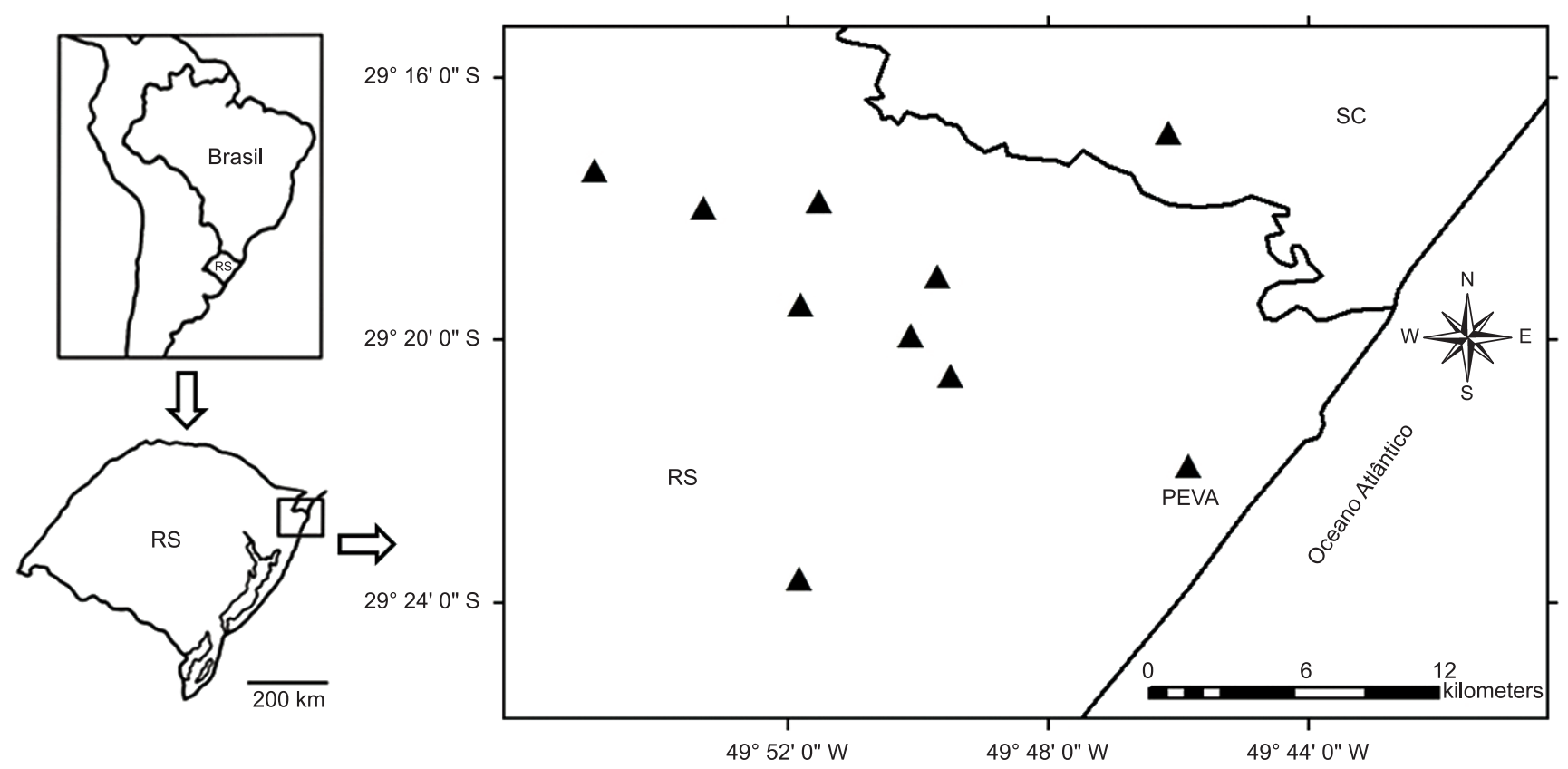

Figura 1. Mapa com a localização dos dez fragmentos de Mata Paludosa amostrados e do Parque Estadual de Itapeva (PEVA) no Rio Grande do Sul (RS) e Santa Catarina (SC).

Figure 1. Map with the location of the ten sampled Swamp forest fragments and Itapeva State Park (PEVA) in Rio Grande do Sul (RS) and Santa Catarina (SC) States.

anteriormente) no interior e nos arredores tanto do fragmento de Mata Paludosa quanto nas áreas de Mata de Restinga no PEVA. Cada ocasião amostral teve duração de cinco dias, com esforço padronizado em três horas/amostrador (totalizando 360 horas-rede), na qual cinco amostradores munidos de rede entomológica percorreram transecções pré-existentes em variados tipos de ambiente em cada área procurando ativamente por borboletas. Quando avistadas eram capturadas e, se possível, identificadas no local (detalhes deste protocolo de amostragem são encontrados em Paz et al. (2008)). Além disso, foram compilados dados de amostragens assistemáticas desenvolvidas na região pelo sétimo autor durante os anos de 1991 a 2012 com a metodologia de redes entomológicas.

Pelo menos dois exemplares de cada espécie foram coletados como material testemunho, assim como os de difícil identificação em campo. Este material encontra-se depositado na Coleção de Lepidoptera do Departamento de Zoologia da Universidade Federal do Rio Grande do Sul. A identificação dos espécimes foi realizada com base na referida coleção, além de consultas a especialistas em determinados grupos de borboletas. A nomenclatura para as famílias, subfamílias e espécies seguiu Lamas (2004) e Mielke (2005), com exceção de Nymphalidae que seguiu determinação taxonômica de Wahlberg et al. (2009).

\section{Elaboração da lista de espécies}

Para a lista de espécies foram incluídos os registros qualitativos das borboletas encontradas nestas formações da Planície Costeira norte do Rio Grande do Sul. Os registros novos para a Mata Atlântica do Rio Grande do Sul foram obtidos por comparação aos trabalhos citados na introdução deste trabalho e as novas ocorrências para o Estado, além de tais citações, através dos trabalhos mencionados em Iserhard \& Romanowski (2004) e Iserhard et al. (2010a). Para efeitos de comparação com demais trabalhos desenvolvidos na Mata Atlântica do Rio Grande do Sul, a família Riodinidae está inserida na família Lycaenidae.

\section{Resultados e Discussão}

Foram registradas 225 espécies de borboletas nas Matas Paludosas e de Restinga nesta área da Planície Costeira do Sul do Brasil, distribuídas em seis famílias e 19 subfamílias. Deste total, 82 espécies foram compartilhadas entre os dois ambientes; 79 espécies são exclusivas das Matas Paludosas e 64 espécies são exclusivas das Matas de Restinga (Tabela 1). Apesar das Matas Paludosas serem inerentemente ricas, com alta diversidade e neste estudo possuírem mais espécies exclusivas quando comparado com o compartilhado entre os dois ambientes, não se deve deixar de evidenciar o registrado nas Restingas, que apesar de terem possuído um esforço amostral inferior e menos áreas estudadas, também mostraram uma alta quantidade de espécies exclusivas.

Avaliando-se a representatividade de cada família por ambiente e para a região como um todo, o padrão é similar a outros inventários realizados na Mata Atlântica do Rio Grande do Sul: Nymphalidae possui proporcionalmente mais espécies, seguida de Hesperiidae, Lycaenidae+Riodinidae, Pieridae e Papilionidae (Tabela 2). Devese levar em consideração o fato de armadilhas atrativas registrarem espécies de borboletas pertencentes somente a Nymphalidae, mesmo assim, apenas com o uso de redes entomológicas, resultados semelhantes foram encontrados para a Floresta Ombrófila Mista e Campos de Cima da Serra (Iserhard et al. 2010a, Ritter et al. 2011) (Tabela 2). A representatividade de borboletas na Floresta Ombrófila Densa apresentou resultados diferentes, nos quais Hesperiidae possuiu maior riqueza quando comparado a Nymphalidae (Tabela 2). Porém, para as áreas de Mata Atlântica, apesar dos esforços amostrais serem os mesmos, as intensidades amostrais são diferentes: Iserhard \& Romanowski (2004) e Iserhard (2009) realizaram inventários intensificados da fauna de borboletas com redes entomológicas da Floresta Ombrófila Densa bimestralmente ao longo de três anos e Santos et al. (2011) utilizaram armadilhas atrativas em uma transecção nesta mesma região ao longo de cinco meses, enquanto 
Bellaver, J. et al.

Tabela 1. Lista de espécies de borboletas registradas em fragmentos de Mata Paludosa e de Mata de Restinga na Planície Costeira do sul do Brasil. Table 1. Butterfly species list recorded in fragments of Swamp Forest and Restinga Forest in southern Brazil Coastal Plain.

\begin{tabular}{|c|c|c|c|c|}
\hline \multirow{2}{*}{ Famílias/subfamílias } & \multirow{2}{*}{ Tribos } & \multirow{2}{*}{ Espécies } & \multicolumn{2}{|c|}{ Ambientes } \\
\hline & & & Mata paludosa & Mata de Restinga \\
\hline \multicolumn{5}{|l|}{ NYMPHALIDAE $(\mathrm{S}=\mathbf{8 5})$} \\
\hline \multirow[t]{10}{*}{ Heliconiinae ( $\mathrm{S}=10)$} & Acraeini & Actinote mamita (Burmeister, 1861) & $\mathrm{X}$ & $\mathrm{X}$ \\
\hline & & Actinote brylla Oberthür, 1917 * \# & $\mathrm{X}$ & \\
\hline & Heliconiini & Agraulis vanillae maculosa (Stichel, 1908) & & $\mathrm{X}$ \\
\hline & & Dione juno juno (Cramer, 1779) & $\mathrm{X}$ & \\
\hline & & Dryas iulia alcionea (Cramer, 1779) & $\mathrm{X}$ & $\mathrm{X}$ \\
\hline & & Eueides isabella dianasa (Hübner, 1806) & & $\mathrm{X}$ \\
\hline & & Heliconius erato phyllis (Fabricius, 1775) & $\mathrm{X}$ & $\mathrm{X}$ \\
\hline & & Heliconius ethilla narcaea Godart, 1819 & $\mathrm{X}$ & $\mathrm{X}$ \\
\hline & & Heliconius sara apseudes (Hübner, 1813) & $\mathrm{X}$ & \\
\hline & & Philaethria wernickei (Röber, 1906) & $\mathrm{X}$ & $\mathrm{X}$ \\
\hline \multirow[t]{5}{*}{ Limenitidinae $(S=5)$} & Limenitidini & Adelpha hyas (Doyère, [1840]) & $\mathrm{X}$ & \\
\hline & & Adelpha lycorias (Godart, 1824) & $\mathrm{X}$ & $\mathrm{X}$ \\
\hline & & Adelpha mythra (Godart, 1824) & & $\mathrm{X}$ \\
\hline & & Adelpha syma (Godart, 1824) & & $\mathrm{X}$ \\
\hline & & Adelpha thessalia indefecta Fruhstorfer, 1913 & & $\mathrm{X}$ \\
\hline \multirow[t]{3}{*}{ Cyrestinae $(\mathrm{S}=3)$} & Cyrestini & Marpesia chiron (Fabricius, 1775) & & $\mathrm{X}$ \\
\hline & & Marpesia zerynthia zerynthia Hübner, [1823] & $\mathrm{X}$ & \\
\hline & & Marpesia petreus (Cramer, 1776) & $\mathrm{X}$ & $\mathrm{X}$ \\
\hline \multirow[t]{12}{*}{ Nymphalinae $(\mathrm{S}=12)$} & Victorinini & Anartia amathea roeselia (Eschscholtz, 1821) & $\mathrm{X}$ & $\mathrm{X}$ \\
\hline & & Anartia jatrophae (Linnaeus, 1763) & $\mathrm{X}$ & $\mathrm{X}$ \\
\hline & Junoniini & Junonia evarete (Cramer, 1779) & $\mathrm{X}$ & $\mathrm{X}$ \\
\hline & Melitaeini & Eresia lansdorfi (Godart, 1819) & $\mathrm{X}$ & $\mathrm{X}$ \\
\hline & & Ortilia ithra (Kirby, 1800) & $\mathrm{X}$ & $\mathrm{X}$ \\
\hline & & Tegosa claudina (Eschscholtz, 1821) & $\mathrm{X}$ & $\mathrm{X}$ \\
\hline & Nymphalini & Colobura dirce dirce (Linnaeus, 1758) & $\mathrm{X}$ & $\mathrm{X}$ \\
\hline & & Hypanartia bella (Fabricius, 1793) & $\mathrm{X}$ & \\
\hline & & Smyrna blomfildia blomfildia (Fabricius, 1781) & $\mathrm{X}$ & \\
\hline & & Vanessa braziliensis (Moore, 1883) & & $\mathrm{X}$ \\
\hline & & Vanessa myrinna (Doubleday, 1849) & $\mathrm{X}$ & \\
\hline & Coeini & Historis acheronta acheronta (Fabricius, 1775) & $\mathrm{X}$ & \\
\hline \multirow[t]{8}{*}{ Biblidinae $(\mathrm{S}=8)$} & Ageroniini & Hamadryas epinome (Felder \& Felder, 1867) & $\mathrm{X}$ & \\
\hline & & Hamadryas februa februa (Hübner, 1823) & $\mathrm{X}$ & $\mathrm{X}$ \\
\hline & Callicorini & $\begin{array}{l}\text { Diaethria clymena meridionalis (H. W. Bates, } \\
\text { 1864) }\end{array}$ & $\mathrm{X}$ & \\
\hline & & Haematera pyrame pyrame (Hübner, [1819]) & $\mathrm{X}$ & \\
\hline & Epicaliini & Catonephele numilia penthia (Hewitson, 1852) & $\mathrm{X}$ & $\mathrm{X}$ \\
\hline & Epiphilini & Epiphile orea orea (Hübner, 1823) & $\mathrm{X}$ & \\
\hline & & Temenis laothoe meridionalis Ebert, 1961 & $\mathrm{X}$ & $\mathrm{X}$ \\
\hline & Eubagini & Dynamine myrrhina (Doubleday, 1849) & & $\mathrm{X}$ \\
\hline \multirow[t]{7}{*}{ Charaxinae $(S=7)$} & Anaeini & Memphis editha (Comstock, 1961) & $\mathrm{X}$ & \\
\hline & & Memphis moruus stheno (Prittwitz, 1865) & $\mathrm{X}$ & $\mathrm{X}$ \\
\hline & & Zaretis strigosus (Gmelin, [1790]) & $\mathrm{X}$ & $\mathrm{X}$ \\
\hline & Preponini & Archaeoprepona chalciope (Hübner, 1823) & $\mathrm{X}$ & $\mathrm{X}$ \\
\hline & & $\begin{array}{l}\text { Archaeoprepona demophon thalpius (Hübner, } \\
\text { 1814) }\end{array}$ & $\mathrm{X}$ & \\
\hline & & Archaeoprepona demophoon (Hübner, 1814) & $\mathrm{X}$ & $\mathrm{X}$ \\
\hline & & Consul fabius drurii (Butler, 1874) & $\mathrm{X}$ & $\mathrm{X}$ \\
\hline \multirow[t]{6}{*}{ Satyrinae $(\mathrm{S}=29)$} & Satyrini & Capronnieria galesus (Godart, 1824) & $\mathrm{X}$ & \\
\hline & & Carminda paeon (Godart, 1824) & $\mathrm{X}$ & $\mathrm{X}$ \\
\hline & & Godartiana muscosa (Butler, 1870) & $\mathrm{X}$ & \\
\hline & & Moneuptychia soter (Butler, 1877) & $\mathrm{X}$ & \\
\hline & & Yphthimoides celmis (Godart, 1824) & & $\mathrm{X}$ \\
\hline & & Forsterinaria necys (Godart, 1824) & $\mathrm{X}$ & $\mathrm{X}$ \\
\hline
\end{tabular}

S, riqueza de espécies; * indica registros novos para a Mata Atlântica do Rio Grande do Sul; \# indica registros novos para o Rio Grande do Sul.

S, species richness; * new records for Rio Grande do Sul Atlantic Forest; \# new records for Rio Grande do Sul State. 
Tabela 1. Continuação...

\begin{tabular}{|c|c|c|c|c|}
\hline \multirow{2}{*}{ Famílias/subfamílias } & \multirow{2}{*}{ Tribos } & \multirow{2}{*}{ Espécies } & \multicolumn{2}{|c|}{ Ambientes } \\
\hline & & & Mata paludosa & Mata de Restinga \\
\hline & & Guaianaza pronophila (Butler, 1867) & & $\mathrm{X}$ \\
\hline & & Hermeuptychia atalanta (Butler, 1867) * \# & $\mathrm{X}$ & $\mathrm{X}$ \\
\hline & & Hermeuptychia gisella (Hayward, 1957) * \# & $\mathrm{X}$ & \\
\hline & & Praepedaliodes phanias (Hewitson, 1862) & $\mathrm{X}$ & $\mathrm{X}$ \\
\hline & & Paryphthimoides phronius (Godart, 1824) & $\mathrm{X}$ & $\mathrm{X}$ \\
\hline & & Paryphthimoides poltys (Prittwitz, 1865) & $\mathrm{X}$ & \\
\hline & & Paryphthimoides grimon (Godart, 1824) & $\mathrm{X}$ & \\
\hline & & Taygetis ypthima Hübner, 1821 & $\mathrm{X}$ & \\
\hline & Brassolini & Blepolenis catharinae (Stichel, 1902) & $\mathrm{X}$ & $\mathrm{X}$ \\
\hline & & Blepolenis batea (Hübner, [1821]) * \# & $\mathrm{X}$ & \\
\hline & & Brassolis sophorae vulpeculus Stichel, 1902 * & $\mathrm{X}$ & \\
\hline & & Caligo brasiliensis (C. Felders, 1862) & $\mathrm{X}$ & \\
\hline & & Caligo martia (Godart, 1824) & $\mathrm{X}$ & $\mathrm{X}$ \\
\hline & & Catoblepia amphirhoe (Hübner, 1825) & $\mathrm{X}$ & \\
\hline & & Dasyophthalma creusa creusa (Hübner, [1821]) & $\mathrm{X}$ & $\mathrm{X}$ \\
\hline & & Dynastor darius darius (Fabricius, 1775) & $\mathrm{X}$ & \\
\hline & & Eryphanis reevesii (Doubleday, 1849) & $\mathrm{X}$ & \\
\hline & & Narope cyllastros Doubleday, 1849 & & $\mathrm{X}$ \\
\hline & & Opoptera sulcius (Staudinger, 1887) & $\mathrm{X}$ & \\
\hline & & Opsiphanes cassiae (Linnaeus, 1758) * \# & $\mathrm{X}$ & \\
\hline & & Opsiphanes quiteria quiteria (Stoll, 1780) & $\mathrm{X}$ & \\
\hline & & Opsiphanes invirae remoliatus Fruhstorfer, 1907 & $\mathrm{X}$ & $\mathrm{X}$ \\
\hline & Morphini & Morpho epistrophus catenaria (Perry, 1811) & $\mathrm{X}$ & \\
\hline \multirow[t]{2}{*}{ Apaturinae $(S=2)$} & & Doxocopa kallina (Staudinger, 1886) & $\mathrm{X}$ & $\mathrm{X}$ \\
\hline & & Doxocopa laurentia (Godart, [1824]) & & $\mathrm{X}$ \\
\hline \multirow[t]{9}{*}{ Danainae $(\mathrm{S}=9)$} & Danaini & Danaus gilippus gilippus (Cramer, 1775) & $\mathrm{X}$ & $\mathrm{X}$ \\
\hline & & Danaus erippus (Cramer, 1775) & $\mathrm{X}$ & $\mathrm{X}$ \\
\hline & & Lycorea halia (Hübner, 1816) & $\mathrm{X}$ & \\
\hline & Ithomiini & Dircenna dero (Hübner, 1823) & $\mathrm{X}$ & \\
\hline & & Episcada carcinia Schaus, 1902 & & $\mathrm{X}$ \\
\hline & & Episcada hymenaea hymenaea (Prittwitz, 1865) & $\mathrm{X}$ & $\mathrm{X}$ \\
\hline & & Mechanitis lisymnia lisymnia (Fabricius, 1793) & $\mathrm{X}$ & $\mathrm{X}$ \\
\hline & & Methona themisto (Hübner, 1818) & & $\mathrm{X}$ \\
\hline & & $\begin{array}{l}\text { Placidina euryanassa C. Felder \& R. Felder, } \\
1860\end{array}$ & & $\mathrm{X}$ \\
\hline \multicolumn{5}{|l|}{ PIERIDAE $(S=14)$} \\
\hline \multirow[t]{9}{*}{ Coliadinae $(\mathrm{S}=9)$} & & Eurema albula sinoe (Godart, 1819) & $\mathrm{X}$ & $\mathrm{X}$ \\
\hline & & Pyrisitia leuce leuce (Boisduval, 1836) & $\mathrm{X}$ & \\
\hline & & Pyrisitia nise tenella (Boisduval, 1836) & $\mathrm{X}$ & \\
\hline & & Eurema elathea (Cramer, 1777) & $\mathrm{X}$ & $\mathrm{X}$ \\
\hline & & Phoebis argante argante (Fabricius, 1775) & $\mathrm{X}$ & $\mathrm{X}$ \\
\hline & & Phoebis neocypris neocypris (Hübner, [1823]) & $\mathrm{X}$ & $\mathrm{X}$ \\
\hline & & Phoebis philea philea (Linnaeus, 1763) & & $\mathrm{X}$ \\
\hline & & Phoebis sennae marcellina (Cramer, 1777) & & $\mathrm{X}$ \\
\hline & & Rhabdodryas trite banksi Breyer, 1939 & & $\mathrm{X}$ \\
\hline \multirow[t]{2}{*}{ Pierinae $(\mathrm{S}=2)$} & Pierini & Ascia monuste orseis (Godart, 1819) & $\mathrm{X}$ & \\
\hline & Antocharidini & Hesperocharis paranensis Schaus, 1898 & & $\mathrm{X}$ \\
\hline \multirow[t]{3}{*}{ Dismorphiinae $(\mathrm{S}=3)$} & & Dismorphia astyocha Hübner, 1831 & $\mathrm{X}$ & \\
\hline & & Dismorphia crisia crisia (Drury, 1782) & $\mathrm{X}$ & \\
\hline & & Enantia melite (Linnaeus, 1763) & & $\mathrm{X}$ \\
\hline \multicolumn{5}{|l|}{ PAPILIONIDAE $(S=10)$} \\
\hline \multirow[t]{4}{*}{ Papilioninae $(\mathrm{S}=10)$} & Troidini & Battus polydamas polydamas (Linnaeus, 1758) & & $\mathrm{X}$ \\
\hline & & Parides agavus (Drury, 1782) & $\mathrm{X}$ & $\mathrm{X}$ \\
\hline & & Parides anchises nephalion (Godart, 1819) & $\mathrm{X}$ & \\
\hline & & Parides bunichus perrhebus (Boisduval, 1836) & & $\mathrm{X}$ \\
\hline
\end{tabular}

S, riqueza de espécies; * indica registros novos para a Mata Atlântica do Rio Grande do Sul; \# indica registros novos para o Rio Grande do Sul. S, species richness; * new records for Rio Grande do Sul Atlantic Forest; \# new records for Rio Grande do Sul State. 
Bellaver, J. et al

Tabela 1. Continuação...

\begin{tabular}{|c|c|c|c|c|}
\hline \multirow{2}{*}{ Famílias/subfamílias } & \multirow{2}{*}{ Tribos } & \multirow{2}{*}{ Espécies } & \multicolumn{2}{|c|}{ Ambientes } \\
\hline & & & Mata paludosa & Mata de Restinga \\
\hline & Papilionini & Heraclides anchisiades capys (Hübner, 1809) & $\mathrm{X}$ & $\mathrm{X}$ \\
\hline & & Heraclides astyalus astyalus (Godart, 1819) & & $\mathrm{X}$ \\
\hline & & Heraclides hectorides (Esper, 1794) & & $\mathrm{X}$ \\
\hline & & $\begin{array}{l}\text { Heraclides thoas brasiliensis (Rothschild \& } \\
\text { Jordan, 1906) }\end{array}$ & $\mathrm{X}$ & $\mathrm{X}$ \\
\hline & & $\begin{array}{l}\text { Pterourus scamander scamander (Boisduval, } \\
\text { 1836) }\end{array}$ & $\mathrm{X}$ & $\mathrm{X}$ \\
\hline & Leptocircini & Protesilaus helios (Rothschild \& Jordan, 1906) & $\mathrm{X}$ & $\mathrm{X}$ \\
\hline \multicolumn{5}{|l|}{ LYCAENIDAE (S = 35) } \\
\hline \multirow[t]{33}{*}{ Theclinae $(\mathrm{S}=33)$} & Eumaeini & Arawacus meliboeus (Fabricius, 1793) & $\mathrm{X}$ & $\mathrm{X}$ \\
\hline & & Arawacus separata (Lathy, 1926) & $\mathrm{X}$ & $\mathrm{X}$ \\
\hline & & Arcas imperialis (Cramer, 1775) *\# & $\mathrm{X}$ & \\
\hline & & Aubergina vanessoides (Prittwitz, 1865) & & $\mathrm{X}$ \\
\hline & & Calycopis caulonia (Hewitson, 1877) & $\mathrm{X}$ & $\mathrm{X}$ \\
\hline & & Chlorostrymon simaethis (Drury, 1773) * & $\mathrm{X}$ & $\mathrm{X}$ \\
\hline & & Cyanophrys acaste (Prittwitz, 1865) & $\mathrm{X}$ & $\mathrm{X}$ \\
\hline & & Dicya dicaea (Hewitson, 1874) & $\mathrm{X}$ & \\
\hline & & Enos thara (Hewitson, 1867) *\# & $\mathrm{X}$ & $\mathrm{X}$ \\
\hline & & Janthecla aurora $($ H. H. Druce, 1907) * \# & $\mathrm{X}$ & \\
\hline & & Janthecla flosculus (H. H. Druce, 1907) & $\mathrm{X}$ & \\
\hline & & Ocaria ocrisia (Hewitson, 1868) & $\mathrm{X}$ & $\mathrm{X}$ \\
\hline & & Ocaria thales (Fabricius, 1793) & $\mathrm{X}$ & $\mathrm{X}$ \\
\hline & & Ministrymon azia (Hewitson, 1873) * \# & & $\mathrm{X}$ \\
\hline & & Nicolaea $\mathrm{sp}$ & $\mathrm{X}$ & \\
\hline & & Nicolaea cupa $($ Druce, 1907$) * \#$ & & $\mathrm{X}$ \\
\hline & & Ostrinotes empusa (Hewitson, 1867$) * \#$ & $\mathrm{X}$ & $\mathrm{X}$ \\
\hline & & Parrhasius orgia (Hewitson, 1867) & & $\mathrm{X}$ \\
\hline & & Pseudolycaena marsyas (Linnaeus, 1758) & $\mathrm{X}$ & $\mathrm{X}$ \\
\hline & & Strephonota tephraeus (Geyer, 1837) & $\mathrm{X}$ & \\
\hline & & Rekoa palegon (Cramer, 1780) & & $\mathrm{X}$ \\
\hline & & Siderus philinna (Hewitson, 1868) & $\mathrm{X}$ & $\mathrm{X}$ \\
\hline & & Strymon astiocha (Prittwitz, 1865) *\# & & $\mathrm{X}$ \\
\hline & & Strymon bazochii (Godart, 1824) & $\mathrm{X}$ & $\mathrm{X}$ \\
\hline & & Strymon dindus (Fabricius, 1793)* \# & & $\mathrm{X}$ \\
\hline & & Strymon eurytulus (Hübner, [1819]) & & $\mathrm{X}$ \\
\hline & & Strymon lucena (Hewitson, 1868) & $\mathrm{X}$ & $\mathrm{X}$ \\
\hline & & Strymon oreala (Hewitson, 1868) & & $\mathrm{X}$ \\
\hline & & Strymon rana (Schaus, 1902) & & $\mathrm{X}$ \\
\hline & & Strymon ziba (Hewitson, 1868) * & $\mathrm{X}$ & $\mathrm{X}$ \\
\hline & & Theritas hemon (Cramer, 1775) & & $\mathrm{X}$ \\
\hline & & Tmolus echion (Linnaeus, 1767) & $\mathrm{X}$ & $\mathrm{X}$ \\
\hline & & Ziegleria hesperitis (Butler \& Druce, 1872) * \# & & $\mathrm{X}$ \\
\hline \multirow[t]{2}{*}{ Polyommatinae $(\mathrm{S}=2)$} & & Leptotes cassius (Cramer, 1775) & & $\mathrm{X}$ \\
\hline & & Hemiargus hanno (Stoll, 1790) * & $\mathrm{X}$ & \\
\hline \multicolumn{5}{|l|}{ RIODINIDAE (S = 15) } \\
\hline \multirow[t]{3}{*}{ Euselasiinae $(\mathrm{S}=3)$} & Euselasiini & Euselasia euploea (Hewitson, 1855) & & $\mathrm{X}$ \\
\hline & & Euselasia hygenius occulta Stichel, 1919 & & $\mathrm{X}$ \\
\hline & & $\begin{array}{l}\text { Euselasia thucydides thucydides (Fabricius, } \\
1793) * \#\end{array}$ & & $\mathrm{X}$ \\
\hline \multirow[t]{6}{*}{ Riodininae $(\mathrm{S}=12)$} & Nymphidiini & Aricoris constantius (Fabricius, 1793$) * \#$ & $\mathrm{X}$ & \\
\hline & & Calospila apotheta (H. W. Bates, 1868) & $\mathrm{X}$ & \\
\hline & & Menander menander $($ Stoll, 1780$) * \#$ & $\mathrm{X}$ & \\
\hline & & Synargis regulus (Fabricius, 1793) * \# & $\mathrm{X}$ & $\mathrm{X}$ \\
\hline & Incertae sedis & Emesis fastidiosa Ménétriés, 1855 * \# & & $\mathrm{X}$ \\
\hline & Symmachiini & Mesene pyrippe Hewitson, 1874 & $\mathrm{X}$ & \\
\hline
\end{tabular}

S, riqueza de espécies; * indica registros novos para a Mata Atlântica do Rio Grande do Sul; \# indica registros novos para o Rio Grande do Sul. S, species richness; * new records for Rio Grande do Sul Atlantic Forest; \# new records for Rio Grande do Sul State. 
Tabela 1. Continuação...

\begin{tabular}{|c|c|c|c|c|}
\hline \multirow{2}{*}{ Famílias/subfamílias } & \multirow{2}{*}{ Tribos } & \multirow{2}{*}{ Espécies } & \multicolumn{2}{|c|}{ Ambientes } \\
\hline & & & Mata paludosa & Mata de Restinga \\
\hline & Mesosemiini & Mesosemia odice (Godart, [1824]) & $\mathrm{X}$ & $\mathrm{X}$ \\
\hline & & Ithomiola nepos (Fabricius, 1793) & $\mathrm{X}$ & \\
\hline & & Ionotus alector (Geyer, 1837) *\# & & $\mathrm{X}$ \\
\hline & Riodinini & Lasaia agesilas (Latreille, 1809) & & $\mathrm{X}$ \\
\hline & & Rhetus periander (Cramer, 1777) *\# & $\mathrm{X}$ & \\
\hline & & Charis cadytis Hewitson, 1866 & $\mathrm{X}$ & \\
\hline \multicolumn{5}{|l|}{ HESPERIIDAE (S = 66) } \\
\hline \multirow[t]{32}{*}{ Pyrginae $(\mathrm{S}=32)$} & Pyrgini & Achlyodes busirus rioja Evans, 1953 & $\mathrm{X}$ & $\mathrm{X}$ \\
\hline & & Achlyodes mithridates thraso (Hübner, [1807]) & $\mathrm{X}$ & $\mathrm{X}$ \\
\hline & & Aethilla echina coracina Butler, 1870 & $\mathrm{X}$ & \\
\hline & & Antigonus liborius areta Evans, 1953 & & $\mathrm{X}$ \\
\hline & & Gesta gesta (Herrich-Schäffer, 1863) & & $\mathrm{X}$ \\
\hline & & Gorgithion begga begga (Prittwitz, 1868) & $\mathrm{X}$ & $\mathrm{X}$ \\
\hline & & Gorgythion beggina escalophoides Evans, 1953 & $\mathrm{X}$ & \\
\hline & & Heliopetes arsalte (Linnaeus, 1758) & & $\mathrm{X}$ \\
\hline & & Heliopetes omrina (Butler, 1870) & $\mathrm{X}$ & \\
\hline & & Milanion leucaspis (Mabille, 1878) & $\mathrm{X}$ & $\mathrm{X}$ \\
\hline & & Nisoniades bipuncta (Schaus, 1902) & $\mathrm{X}$ & $\mathrm{X}$ \\
\hline & & Pyrgus orcus (Stoll, 1780) & $\mathrm{X}$ & $\mathrm{X}$ \\
\hline & & Pythonides lancea (Hewitson, 1868) & $\mathrm{X}$ & \\
\hline & & Quadrus cerialis (Stoll, 1782) & $\mathrm{X}$ & \\
\hline & & $\begin{array}{l}\text { Trina geometrina geometrina (C.Felder \& } \\
\text { R.Felder, 1867) }\end{array}$ & $\mathrm{X}$ & \\
\hline & & Xenophanes tryxus (Stoll, 1780) & $\mathrm{X}$ & \\
\hline & & Zera tetrastigma erisichton (Plötz, 1884) * & & $\mathrm{X}$ \\
\hline & Eudamini & Astraptes aulus (Plötz, 1881) & $\mathrm{X}$ & $\mathrm{X}$ \\
\hline & & Astraptes fulgerator fulgerator (Walch, 1775) & $\mathrm{X}$ & $\mathrm{X}$ \\
\hline & & Autochton zarex (Hübner, 1818) & & $\mathrm{X}$ \\
\hline & & Celaenorrhinus similis Hayward, 1933 & $\mathrm{X}$ & $\mathrm{X}$ \\
\hline & & Epargyreus exadeus exadeus (Crammer, 1779) & $\mathrm{X}$ & \\
\hline & & Nascus phocus (Crammer, 1777) & $\mathrm{X}$ & \\
\hline & & Urbanus albimargo (Mabille, 1875) & $\mathrm{X}$ & \\
\hline & & Urbanus dorantes (Stoll, 1790) & $\mathrm{X}$ & $\mathrm{X}$ \\
\hline & & Urbanus esta Evans, 1952 & $\mathrm{X}$ & $\mathrm{X}$ \\
\hline & & Urbanus procne (Plötz, 1880) & $\mathrm{X}$ & $\mathrm{X}$ \\
\hline & & Urbanus simplicius (Stoll, 1790) & & $\mathrm{X}$ \\
\hline & & Urbanus teleus (Hübner, 1821) & $\mathrm{X}$ & $\mathrm{X}$ \\
\hline & Pyrrhopygini & Elbella lamprus (Hopffer, 1874) *\# & $\mathrm{X}$ & \\
\hline & & Mysoria barcastus barta Evans, 1951* & & $\mathrm{X}$ \\
\hline & & Pyrrhopyge sp. & $\mathrm{X}$ & $\mathrm{X}$ \\
\hline \multirow[t]{14}{*}{ Hesperiinae $(\mathrm{S}=34)$} & & Ancyloxypha nitedula (Burmeister, 1878) & $\mathrm{X}$ & \\
\hline & & Appia appia Evans, 1955 & $\mathrm{X}$ & \\
\hline & & Arita arita (Schaus, 1902) * & $\mathrm{X}$ & \\
\hline & & Callimormus interpunctata (Plötz, 1884) & & $\mathrm{X}$ \\
\hline & & Callimormus rivera (Plötz, 1882) & $\mathrm{X}$ & $\mathrm{X}$ \\
\hline & & Chalcone santarus (Bell, 1940) & $\mathrm{X}$ & $\mathrm{X}$ \\
\hline & & Calpodes ethlius (Stoll, 1782) & & $\mathrm{X}$ \\
\hline & & Conga chydaea (Butler, 1877) & $\mathrm{X}$ & $\mathrm{X}$ \\
\hline & & Corticea corticea (Plötz, 1882) & $\mathrm{X}$ & \\
\hline & & Cymaenes gisca Evans, 1955 & & $\mathrm{X}$ \\
\hline & & $\begin{array}{l}\text { Cymaenes tripunctus theogenis (Capronnier, } \\
\text { 1874) }\end{array}$ & & $\mathrm{X}$ \\
\hline & & Cymaenes lepta (Hayward, 1939) & & $\mathrm{X}$ \\
\hline & & Hylephila phyleus (Drury, 1773) & $\mathrm{X}$ & \\
\hline & & Lychnuchoides ozias ozias (Hewitson, 1878) & $\mathrm{X}$ & $\mathrm{X}$ \\
\hline
\end{tabular}

S, riqueza de espécies; * indica registros novos para a Mata Atlântica do Rio Grande do Sul; \# indica registros novos para o Rio Grande do Sul. S, species richness; * new records for Rio Grande do Sul Atlantic Forest; \# new records for Rio Grande do Sul State. 
Tabela 1. Continuação...

\begin{tabular}{|c|c|c|c|c|}
\hline \multirow{2}{*}{ Famílias/subfamílias } & \multirow{2}{*}{ Tribos } & \multirow{2}{*}{ Espécies } & \multicolumn{2}{|c|}{ Ambientes } \\
\hline & & & Mata paludosa & Mata de Restinga \\
\hline & & Lychnuchus celsus (Fabricius, 1793) & & $\mathrm{X}$ \\
\hline & & $\begin{array}{l}\text { Miltomiges cinnamomea (Herrich-Schäffer, } \\
1869 \text { ) }\end{array}$ & & $\mathrm{X}$ \\
\hline & & Mnasilus allubita (Butler, 1870) & $\mathrm{X}$ & \\
\hline & & Morys geisa geisa (Möschler, 1879) * \# & & $\mathrm{X}$ \\
\hline & & Nastra lurida (Herrich-Schäffer, 1869) & & $\mathrm{X}$ \\
\hline & & Nyctelius nyctelius nyctelius (Latreille, [1824]) & $\mathrm{X}$ & $\mathrm{X}$ \\
\hline & & Panoquina ocola ocola (W. H. Edwards, 1863) & $\mathrm{X}$ & \\
\hline & & Parphorus pseudecorus (Hayward, 1934) & $\mathrm{X}$ & \\
\hline & & Perichares philetes aurina Evans, 1955 & $\mathrm{X}$ & \\
\hline & & Polites vibex catilina (Plötz, 1886) & & $\mathrm{X}$ \\
\hline & & Pompeius dares (Plötz, 1883) * \# & & $\mathrm{X}$ \\
\hline & & Pompeius pompeius (Latreillle, [1824]) & $\mathrm{X}$ & \\
\hline & & Saliana longirostris (Sepp, [1840]) & $\mathrm{X}$ & \\
\hline & & Talides riosa Evans, $1955 * \#$ & $\mathrm{X}$ & \\
\hline & & Vehilius inca (Scudder, 1872) & $\mathrm{X}$ & \\
\hline & & Vehilius stictomenes stictomenes (Butler, 1877) & & $\mathrm{X}$ \\
\hline & & Vettius phyllus prona Evans, $1955 *$ & $\mathrm{X}$ & $\mathrm{X}$ \\
\hline & & Virga austrinus (Hayward, 1934) * & $\mathrm{X}$ & \\
\hline & & Zariaspes mys (Hübner, [1808]) & & $\mathrm{X}$ \\
\hline & & Wallengrenia premnas (Wallengren, 1860) * & & $\mathrm{X}$ \\
\hline S Total $=225$ & & & 161 & 146 \\
\hline
\end{tabular}

S, riqueza de espécies; * indica registros novos para a Mata Atlântica do Rio Grande do Sul; \# indica registros novos para o Rio Grande do Sul.

S, species richness; * new records for Rio Grande do Sul Atlantic Forest; \# new records for Rio Grande do Sul State.

Tabela 2. Representatividade (\%) das famílias de borboletas em diferentes fisionomias da Mata Atlântica do Rio Grande do Sul, Brasil.

Table 2. Percentage of composition of butterfly families in different phytophysiognomies of Atlantic Forest in Rio Grande do Sul State, Brazil.

\begin{tabular}{lccccc}
\hline & PCN & MPA & RES & FOD $^{\mathbf{1 , 2 , 3}}$ & FOM $^{\mathbf{3}, \mathbf{4 , 5}, \mathbf{6}, \mathbf{7}}$ \\
\hline Nymphalidae & 37,8 & 43,5 & 34,8 & 30,0 & 38,8 \\
Hesperiidae & 29,3 & 28,0 & 28,1 & 35,7 & 28,2 \\
Lycaenidae + Riodinidae & 22,3 & 19,2 & 24,7 & 23,6 & 19,4 \\
Pieridae & 6,2 & 5,6 & 6,2 & 7,3 & 8,7 \\
Papilionidae & 4,4 & 3,7 & 6,2 & 3,4 & 4,9 \\
\hline
\end{tabular}

PCN = Planície Costeira Norte; MPA = Mata Paludosa; RES = Restinga; FOD $=$ Floresta Ombrófila Densa; FOM $=$ Floresta Ombrófila Mista. $\mathrm{PCN}=$ North Coastal Plain; MPA $=$ Swamp Forest; RES $=$ Restinga Forest; FOD = Subtropical Atlantic Forest; FOM = Araucaria Moist Forest. ${ }^{1}$ Iserhard \& Romanwoski (2004); ${ }^{2}$ Iserhard (2009); ${ }^{3}$ Santos et al. (2011); ${ }^{4}$ Iserhard et al. (2010a); ${ }^{5}$ Romanowski et al. (2009); ${ }^{6}$ Pedrotti et al. (2011); ${ }^{7}$ Ritter et al. (2011).

que para a Floresta Ombrófila Mista foram apenas dois anos de amostragem com rede entomológica (Iserhard et al. 2010a) e dois anos de amostragem com armadilhas atrativas (Santos et al. 2011). Em geral, ambientes de Mata Atlântica se aproximam de uma boa representatividade de todos os grupos e famílias de borboletas quando Hesperiidae ultrapassa Nymphalidae em espécies (Brown \& Freitas 2000b), como demonstrado na compilação dos trabalhos de Iserhard \& Romnowski (2004), Iserhard (2009) e Santos et al. (2011). Este padrão é encontrado, também, em outros inventários na Mata Atlântica brasileira (Brown 1992, Mielke \& Casagrande 1998, Brown \& Freitas 2000a, b, Santos et al. 2008, Dolibaina et al. 2011, Francini et al. 2011). Mesmo que este inventário tenha obtido um alto esforço amostral com armadilhas para a captura exclusiva de borboletas frugívoras da família Nymphalidae, sugere-se que um aumento no esforço amostral no presente estudo provavelmente levaria a uma maior representatividade de Hesperiidae que superaria Nymphalidae em número de espécies, já que em Hesperiidae o acréscimo de novas espécies é mais lento e constante ao longo do tempo (Iserhard 2009) e a diferença entre tais famílias é de apenas 19 espécies.

Foram encontrados 25 registros não publicados para o Rio Grande do Sul e 35 novos registros de borboletas para o bioma Mata Atlântica neste Estado, sendo seis Nymphalidae, dez Hesperiidae, doze Lycaenidae e sete Riodinidae (Tabela 1), ressaltando a importância da realização de inventários em lacunas de conhecimento como as terras baixas na Planície Costeira do Brasil Meridional. Alguns destes registros chamam a atenção pela forte associação com os hábitats onde foram observadas: Actinote brylla Oberthür, 1917 e Memphis editha (Comstock, 1961) são espécies características de ambientes de terras baixas mais próximas a Mata Atlântica de faixas litorâneas. Actinote brylla, Memphis editha, Aricoris constantius (Fabricius, 1793) e Euselasia thucydides thucydides (Fabricius, 1793) tiveram seus limites sul de distribuição ampliados para a Mata Atlântica brasileira, anteriormente registradas até Santa Catarina (Callaghan 2001, 2010, Dias et al. 2012). É importante ressaltar a quantidade de riodinídeos exclusivos para estas formações de Mata Atlântica, bem como o primeiro registro de Rethus periander (Cramer, 1777) e do licenídeo Arcas imperialis (Cramer, 1775) para o Rio Grande do Sul. A primeira espécie foi encontrada apenas uma vez e a segunda espécie foi registrada duas vezes, sendo que ambas estavam associadas ao interior da mata paludosa na parte mais preservada do fragmento do Parque Estadual de Itapeva.

Inventários são fundamentais para o conhecimento e para a conservação tanto dos táxons estudados quanto dos ambientes aos quais eles estão associados, pois podem revelar características importantes de comunidades locais, tais como a utilização de recursos e a preferência de habitat, além de contribuir para o conhecimento 
da fauna regional (Marchiori \& Romanowski 2006). Torna-se necessário a divulgação deste tipo de publicação para que estudos ecológicos futuros possam ser adequadamente elaborados e bem conduzidos. A fragmentação e/ou conversão de ambientes nativos em áreas antropizadas pode ser a forma mais destrutiva de perda de habitat (Dunn 2004) e, para Lepidoptera, pode levar à substituição de grupos de espécies especialistas por borboletas generalistas, comuns e adaptadas a ambientes degradados (Brown 1996). As áreas de Mata Paludosa e de Restinga estão fortemente ameaçadas através de duas principais ações antrópicas: agropecuária (cultivo do arroz e manejo de gado) e a construção civil (especulação imobiliária em áreas litorâneas). A paisagem remanescente desses ecossistemas encontra-se restrita a pequenas manchas de floresta e, apesar deste cenário alarmante, são sistemas ainda pouco estudados (Kindel 2002). Em virtude desta devastação, espécies de plantas e animais estão sendo eliminadas, restringindo a diversidade biótica (Maciel 1984), muitas vezes antes de ser conhecida. Este trabalho reforça o direcionamento de esforços de amostragem em regiões pouco conhecidas, visando ampliar as informações sobre a distribuição e a ocorrência de borboletas no Brasil Meridional. O presente estudo pretendeu contribuir com este propósito e sugere que a geração de conhecimento sobre a diversidade de borboletas de uma ampla área na Planície Costeira do sul do Brasil - e em particular, o grande número de novos registros obtidos - contribua para a preservação destes ambientes, ainda tão pobremente representados em Unidades de Conservação e crescentemente ameaçados pela pressão antrópica.

\section{Agradecimentos}

Os autores agradecem aos colegas Lucas A. Kaminski, Fabiana Camargo, Eduardo C. Teixeira, Daniel S. Castro, Nicolás Mega, Beatriz B. Aydos, Maury S. L. Abreu, Andressa C. de Castro, Pablo L. Bos, Luiza Moucachen, Patrick Colombo e aos alunos do curso de graduação em Ciências Biológicas da disciplina de Inventário de Fauna e Avaliação da Diversidade da Universidade Federal do Rio Grande do Sul. Aos doutores Ricardo Dobrovolski e Andreas Kindel pelas informações e fornecimento dos mapas da região. Ao Dr. Olaf Mielke, M.Sc. Diego Dolibaina, M.Sc. Fernando Dias, M.Sc. Eduardo de Proença Barbosa, M.Sc. Noemy Seraphim e ao Sr. Curtis Callaghan pelas identificações das borboletas. A SEMA-RS pela autorização de pesquisa $\mathrm{n}^{\mathrm{o}} 325$ e aos administradores do Parque Estadual de Itapeva Patrick Colombo (ano de 2005) e Paulo Grubler (anos de 2010 e 2011). Ao Centro Ecológico litoral Norte Assessoria e Formação em Cultura Ecológica e à Marlene Apolinário pelo apoio e hospedagem e aos proprietários das áreas particulares pela permissão de pesquisa em suas propriedades. Este trabalho foi financiado pela CAPES e pelo edital SISBIOTA - CNPq (RedeLep - Rede Nacional de Pesquisa e Conservação de Lepidópteros). Contribuição n ${ }^{\circ} 562$ do Departamento de Zoologia da Universidade Federal do Rio Grande do Sul.

\section{Referências Bibliográficas}

AGRITEMPO. Sistema de Monitoramento Agrometeorológico. http://www. agritempo.gov.br (último acesso em 18/01/2012).

BOND-BUCKUP, G. 2008. Biodiversidade dos Campos de Cima da Serra. Libretos, Porto Alegre.

BROWN, K.S. 1991. Conservation of Neotropical Environments: Insects as Indicators. In The conservation of insects and their habitats (N.M. Collins \& J.A. Thomas, eds). Academic Press, London, p.350-404.

BROWN, K.S. 1992. Borboletas da Serra do Japi: diversidade, habitats, recursos alimentares e variação temporal. In História natural da Serra do Japi: ecologia e preservação de uma área florestal no sudeste do Brasil (L.P.C. Morellato, ed.). Campinas, p.142-186.
BROWN, K.S. 1996. Diversity of Brazilian Lepidoptera: history of study, methods for measurement, and use as indicator for genetic, specific and system richness. In Biodiversity in Brazil, a first approach (C.E.M. Bicudo \& N.A. Menezes, eds). Instituto de Botânica, CNPq, São Paulo, p.223-253.

BROWN, K.S. 1997. Diversity, disturbance, and sustainable use of neotropical forests: insects as indicators for conservation monitoring. J. Insect Conserv. 1(1):25-42. http://dx.doi.org/10.1023/A:1018422807610

BROWN, K.S. \& FREITAS, A.V.L. 1999. Lepidoptera. In Biodiversidade do Estado de São Paulo, Brasil. Invertebrados Terrestres (C.R.F. Brandão \& E.M. Cancello, eds). FAPESP, São Paulo, p.225-245.

BROWN, K.S. \& FREITAS, A.V.L. 2000a. Diversidade de Lepidoptera em Santa Teresa, Espírito Santo. Bol. Mus. Biol. Mello Leitão 11(12):71-118.

BROWN, K.S. \& FREITAS, A.V.L. 2000b. Atlantic Forest Butterflies: indicators for landscape conservation. Biotropica 32(4b):934-956. http:// dx.doi.org/10.1111/j.1744-7429.2000.tb00631.x

CALLAGHAN, C.J. 2001. New riodinids from the Central Brazilian plateau (Lepidoptera, Riodinidae). Rev. Bras. Zool. 18(3):765-778. http://dx.doi. org/10.1590/S0101-81752001000300013

CALLAGHAN, C.J. 2010. A re-evaluation of the Aricoris constantius group with the recognition of three species (Lepidoptera: Riodinidae). Zoologia 27(3):395-402.

COLOMBO, P., KINDEL, A., VINCIPROVA, G. \& KRAUSE, L. 2008. Composição e ameaças à conservação dos anfíbios anuros do Parque Estadual de Itapeva, município de Torres, Rio Grande do Sul, Brasil. Biota Neotrop. 8(3): http://www.biotaneotropica.org.br/v8n3/pt/ fullpaper?bn01208032008+pt (último acesso em 18/01/2012).

CORSEUIL, E., QUADROS, F.C., TESTON, J.A. \& MOSER, A. 2004. Borboletas (Lepidoptera, Papilionoidea e Hesperioidea) coletadas no Centro de Pesquisas e Conservação da Natureza Pró-Mata. 4. Lycaenidae. Divul. Mus. Ciênc. Tecnol. PUCRS 9:65-70.

DAILY, G.C. \& EHRLICH, P.R. 1995. Preservation of biodiversity in small rainforest patches: rapid evaluations using butterfly trapping. Biodiv. Conserv. 4:35-55. http://dx.doi.org/10.1007/BF00115313

DIAS, F.M.S., CASAGRANDE, M.M. \& MIELKE, O.H.H. 2012 Taxonomic notes on the leafwing butterflies Memphis leonida (Stoll) and Memphis editha (Comstock), stat. nov. (Lepidoptera: Nymphalidae: Charaxinae). Zootaxa 3343:16-30.

DOLIBAINA, D.R., MIELKE, O.H.H. \& CASAGRANDE, M.M. 2011. Borboletas (Papilionoidea e Hesperioidea) de Guarapuava e arredores, Paraná, Brasil: um inventário com base em 63 anos de registros. Biota Neotrop. 11(1): http://www.biotaneotropica.org.br/v11n1/en/ abstract?article+bn00211012011 (ultimo acesso em 18/01/2012).

DUNN, R.R. 2004. Managing the tropical landscape: a comparison of the effects of logging and Forest conversion to agriculture on ants, birds and lepidoptera. Fores. Ecol. Manag. 191:215-224. http://dx.doi. org/10.1016/j.foreco.2003.12.008

FRANCINI, R.B., DUARTE, M., MIELKE, O.H.H., CALDAS, A. \& FREITAS, A.V.L. 2011. Butterflies (Lepidoptera, Papilionoidea e Hesperioidea) of the "Baixada Santista" region, coastal São Paulo, southeastern Brazil. Rev. Bras. Entomol. 55(1): 55-68. http://dx.doi. org/10.1590/S0085-56262011000100010

FREITAS, A.V.L., FRANCINI, R.B. \& BROWN, K.S. 2003. Insetos como indicadores ambientais. In Métodos de estudo em Biologia da Conservação e Manejo da Vida Silvestre (L. Cullen Junior, R. Rudran \& C. Valladares-Pádua, orgs.). Fundação Boticário e Editora da UFPR, Curitiba, p.125-151.

FREITAS, A.V.L., LEAL, J.R., UEHARA-PRADO, M. \& IANNUZZI, L. 2006. Insetos como indicadores de conservação da paisagem. In Biologia da Conservação: Essências (C.F.D. Rocha, H.G. Bergallo, M.V. Sluys \& M.A.S. Alves, eds.). RiMa Editora, São Carlos, 15:357-384.

HUGHES, J.B., GRETCHEN, C.D. \& EHRLICH, P.R. 2000. Conservation of insect diversity: a habitat approach. Conserv. Biol. 14(6):1788-1797. http://dx.doi.org/10.1046/j.1523-1739.2000.99187.x 
ISERHARD, C.A. 2009. Estrutura e composição da assembléia de borboletas (Lepidoptera: Papilionoidea e Hesperioidea) em diferentes formações da Mata Atlântica do Rio Grande do Sul, Brasil. Tese de doutorado, Universidade Federal do Rio Grande do Sul, Porto Alegre.

ISERHARD, C.A. \& ROMANOWSKI, H.P. 2004. Lista de espécies de borboletas (Lepidoptera, Papilionoidea e Hesperioidea) da região do vale do Rio Maquiné, Rio Grande do Sul, Brasil. Rev. Bras. Zool. 21(3):649-662. http://dx.doi.org/10.1590/S0101-81752004000300027

ISERHARD, C.A., QUADROS, M.T., ROMANOWSKI, H.P. \& MENDONÇA-JUNIOR, M.S. 2010a. Borboletas (Lepidoptera: Papilionoidea e Hesperioidea) ocorrentes em diferentes ambientes na Floresta Ombrófila Mista e Campos de Cima da Serra do Rio Grande do Sul, Brasil. Biota Neotrop. 10: www.biotaneotropica.org.br/v10n1/pt/ abstract?article+bn02910012010 (último acesso em 23/12/2011).

ISERHARD, C.A., SILVA, A.K., QUADROS, M.T., CASTRO, D.S. \& ROMANOWSKI, H.P. 2010b. Lepidoptera, Nymphalidae, Heliconiinae, Heliconius sara apseudes (Hübner, 1813): distribution extension. Check List 6:316-318.

KINDEL, A. 2002. Diversidade e estratégias de dispersão de plantas vasculares da floresta paludosa do faxinal, Torres, RS. Tese de doutorado, Universidade Federal do Rio Grande do Sul, Porto Alegre.

LAMAS, G. 2004. Atlas of Neotropical Lepidoptera. Checklist: Part 4A. Hesperioidea \& Papilionoidea. Gainesville, Scientific Publishers.

MACIEL, N.C. 1984. Fauna das restingas do estado do Rio de Janeiro: levantamento histórico. In Restingas: origem, estrutura e processos (L.D. Lacerda, D.S.D. Araújo, R. Cerqueira \& B. Turcq, eds). CEUFF, Niterói, p.227-284.

MALUF, J.R.T. 2000. Nova classificação climática do Estado do Rio Grande do Sul. Rev. Bras. Agrometeorol. 8(1):141-150.

MARCHIORI, M.O. \& ROMANOWSKI, H.P. 2006. Borboletas (Lepidoptera, Papilionoidea e Hesperioidea) do Parque Estadual do Espinilho e entorno, Rio Grande do Sul, Brasil. Rev. Bras. Zool. 23(4):1029-1037. http:// dx.doi.org/10.1590/S0101-81752006000400007

MIELKE, O.H.H. 2005. Catalogue of the American Hesperioidea: Hesperiidae (Lepidoptera). Complementary and supplmentary parts to the checklist of the Neotropical region. Hesperioidea: Hesperiidae: Pyrrhopyginae. Sociedade Brasileira de Zoologia, Curitiba.

MIELKE, O.H.H. \& CASAGRANDE, M.M. 1998. Papilionoidea e Hesperioidea (Lepidoptera) do Parque Estadual do Morro do Diabo, Teodoro Sampaio, São Paulo, Brasil. Rev. Bras. Zool. 14:966-1001.

MORAIS, A.B.B., ROMANOWSKI, H.P., ISERHARD, C.A., MARCHIORI, M.O. \& SEGUI, R. 2007. Mariposas del Sur de Sudamérica (Lepidoptera: Papilionoidea e Hesperioidea). Ciênc. Ambient. 35:29-46.

NEW, T.R. 1997. Are Lepidoptera an effective "umbrella group" for biodiversity conservation? J. Insect Conserv. 1(1):5-12. http://dx.doi. org/10.1023/A:1018433406701

PASCHOAL, M.E.S. \& CAVASSAN, O. 1999. A flora arbórea da mata de brejo do ribeirão do Pelintra, Agudos, SP. Naturalia 24:171-191.

PAZ, A.L.G., ROMANOWSKI, H.P. \& MORAIS, A.B.B. 2008. Nymphalidae, Papilionidae e Pieridae (Lepidoptera: Papilionoidea) da Serra do Sudeste do Rio Grande do Sul, Brasil. Biota Neotrop. 8(1): http://www. biotaneotropica.org.br/v8n1/pt/abstract?Inventory+bn0160801 2008 (ultimo acesso em 18/01/2012).

PEDROTTI, V.S., BARROS, M.P., ROMANOWSKI, H.P. \& ISERHARD, C.A. 2011. Borboletas frugívoras (Lepidoptera: Nymphalidae) ocorrentes em um fragmento de Floresta Ombrófila Mista no Rio Grande do Sul, Brasil. Biota Neotrop. 11(1): www.biotaneotropica.org.br/v11n1/pt/ fullpaper?bn03011012011+pt (ultimo acesso em 18/01/2012).
QUADROS, F.C., DORNELES, A.L. \& CORSEUIL, E. 2004. Ninfalídeos (Lepidoptera, Nymphalidae) ocorrentes no norte da planície costeira do Rio Grande do Sul, Brasil. Bioc. 12(2):147-164.

RAMBO, B. 2005. A fisionomia do Rio Grande do Sul: ensaio de monografia natural. Unisinos, São Leopoldo.

RIBEIRO, M.C., METZGER, J.P., MARTENSEN, A.C., PONZONI, F.J. \& HIROTA, M.M. 2009. The Brazilian Atlantic Forest: How much is left, and how is the remaining forest distributed? Implications for conservation. Biol. Conserv. 142:1141-1153. http://dx.doi.org/10.1016/j. biocon.2009.02.021

RIO GRANDE DO SUL. Secretaria Estadual do Meio Ambiente - SEMA. 2006. Plano de Manejo do Parque Estadual de Itapeva. SEMA, Porto Alegre.

RITTER, C.D., LEMES, R., MORAIS, A.B.B. \& DAMBROS, C.S. 2011. Borboletas (Lepidoptera: Hesperioidea e Papilionoidea) de fragmentos de Floresta Ombrófila Mista, Rio Grande do Sul, Brasil. Biota Neotrop. 11(1): http://www.biotaneotropica.org.br/v11n1/pt/ abstract?article+bn00511012011 (último acesso em 14/01/2012).

ROCHA, C.F.D., BERGALLO, H.G., ALVES, M.A. \& SLUYS, M.V. 2004. A Restinga de Jurubatiba e a conservação dos ambientes de Restinga do Estado do Rio de Janeiro. In Pesquisas de longa duração na restinga de Jurubatiba: Ecologia, história natural e conservação (C.F. Rocha, F.A. Esteves \& F.R. Scarano, orgs.). RIMA, São Carlos.

ROMANOWSKI, H.P., ISERHARD, C.A. \& HARTZ, S.M. 2009. Borboletas da floresta com araucária. In Floresta com araucária: ecologia, conservação e desenvolvimento sustentável (C.R. Fonseca, A.F. Souza, A.M. Leal-Zanchet, T.L. Dutra, A. Backes \& G. Ganade, orgs.). Holos, Ribeirão Preto, p.250-260.

SANTOS, E.C., MIELKE, O.H.H. \& CASAGRANDE, M.M. 2008. Inventários de borboletas no Brasil: estado da arte e modelo de áreas prioritárias para pesquisa com vistas à conservação. Nat. Conserv. 6(2):68-90.

SANTOS, J.P., ISERHARD, C.A., TEIXEIRA, M.O. \& ROMANOWSKI, H.P. 2011. Guia de borboletas frugívoras das Florestas Ombrófilas Densa e Mista do Rio Grande do Sul, Brasil. Biota Neotrop. 11(3): http://www. biotaneotropica.org.br/v11n1/pt/abstract?article+bn03011012011 (ultimo acesso em 14/01/2012).

TESTON, J.A. \& CORSEUIL, E. 1999. Borboletas (Lepidoptera, Rhopalocera) ocorrentes no Centro de Pesquisas e Conservação da Natureza Pró-Mata. 1. Papilionidae. Divul. Mus. Ciênc. Tecnol. PUCRS 4:217-228.

TESTON, J.A. \& CORSEUIL, E. 2000. Borboletas (Lepidoptera, Rhopalocera) ocorrentes no Centro de Pesquisas e Conservação da Natureza Pró-Mata. 2. Pieridae. Divul. Mus. Ciênc. Tecnol. PUCRS 5:143-155.

TESTON, J.A. \& CORSEUIL, E. 2002. Borboletas (Lepidoptera, Rhopalocera) ocorrentes no Centro de Pesquisas e Conservação da Natureza Pró-Mata. 3: Nymphalidae. Divul. Mus. Ciênc. Tecnol. PUCRS 7:1-20.

TONIATO, M.T.Z., LEITÃO-FILHO, H.F. \& RODRIGUES, R.R. 1998. Fitossociologia de um remanescente de floresta higrófila (mata de brejo) em Campinas, SP. Rev. Bras. Bot. 21(2):197-210. http://dx.doi. org/10.1590/S0100-84041998000200012

WAECHTER, J.L. 1985. Aspectos ecológicos da vegetação de restinga do Rio Grande do Sul, Brasil. Comun. Mus. Ciênc. PUCRS, série Bot. 33:49-68.

WAHLBERG, N., LENEVEU, J., KODANDARAMAIAH, U., PEÑA, C., NYLIN, S., FREITAS, A.V.L. \& BROWER, A.V.Z. 2009. Nymphalid butterflies diversity following near demise at the Cretaceous/Tertiary boundary. Proc. R. Soc. London B. Biol. Sci. 276:4295-4302. http:// dx.doi.org/10.1098/rspb.2009.1303 